\title{
THE EXISTENCE OF SURFACES OF CONSTANT MEAN CURVATURE WITH ARBITRARY JORDAN CURVES AS ASSIGNED BOUNDARY ${ }^{1}$
}

\section{HELMUT WERNER}

1. Introduction. In the first half of this century the problem of Plateau was treated for minimal surfaces. In 1931 Douglas [3] solved the problem of Plateau for one given arbitrary Jordan curve. In the following time the result was generalized to more complicated boundaries and to minimal surfaces of higher topological structure. For details we refer to the book of Courant [1] which also contains a bibliography on this subject.

Heinz made the first step in transferring the results on minimal surfaces to the larger class of surfaces of constant mean curvature $H$ in [4]. He proved the existence of such a surface with $|H|$ $<\left(17^{1 / 2}-1\right) / 8$ which is bounded by a rectifiable Jordan curve in the unit sphere. A short statement of this result is also given in [5].

In the meantime the author improved the bound for $|H|$ to $1 / 2$ and enlarged the result to surfaces which have a boundary consisting of more than one rectifiable Jordan curve, [6]. In this note I intend to point out that it is not necessary for the Jordan curve to be rectifiable. We prove the following result.

THEOREM 1. Let $\Gamma$ be a closed Jordan curve contained in the unit sphere $x^{2}+y^{2}+z^{2} \leqq 1$. Then there is a surface of constant mean curvature $H$ provided $|H|<1 / 2$ which has the boundary $\Gamma$.

Here we confine ourselves to this simple case, while in another paper I will discuss the question of multiple connected surfaces and higher topological structure.

The stated Theorem 1 is equivalent to the following analytical statement.

THEOREM $1^{\prime}$. There is a vector function $\mathfrak{x}(u, v)=(x(u, v), y(u, v), z(u, v))$ for $u^{2}+v^{2} \leqq 1$ with the following properties:

(A) It has first and second continuous derivatives in $u^{2}+v^{2}<1$ and satisfies the differential equations

$$
\begin{aligned}
\Delta \mathfrak{x} & =2 H\left(\mathfrak{x}_{u} \times \mathfrak{x}_{\mathfrak{v}}\right) \quad \text { for }|H|<1 / 2, \\
\mathfrak{x}_{u} & =\mathfrak{x}_{v}^{2}, \quad\left(\mathfrak{x}_{u}, \mathfrak{x}_{v}\right)=0 ;
\end{aligned}
$$

Received by the editors March 30, 1959.

${ }^{1}$ This research was supported by the Office of Naval Research, Contract Nonr 228(09). 
(B) the function $\mathfrak{x}(u, v)$ is continuous in $u^{2}+v^{2} \leqq 1$ and the inequality $|\mathfrak{x}| \leqq 1$ holds;

(C) the function $\mathfrak{x}(u, v)$ gives a topological mapping of $u^{2}+v^{2}=1$ onto $\Gamma$.

Here $\left(\mathfrak{x}_{u} \times \mathfrak{x}_{v}\right)$ denotes the vector product

$$
\left(y_{u} z_{v}-y_{v} z_{u}, z_{u} x_{v}-z_{v} x_{u}, x_{u} y_{v}-x_{v} y_{u}\right)
$$

and

$$
\left(\mathfrak{x}_{u}, \mathfrak{x}_{\mathfrak{v}}\right)=x_{u} x_{v}+y_{u} y_{v}+z_{u} z_{v}
$$

the scalar product. We write $\mathfrak{x}_{u}^{2}=\left(\mathfrak{x}_{u}, \mathfrak{x}_{u}\right)$.

We use $D$ to denote the point set $\left\{(u, v) \mid u^{2}+v^{2}<1\right\}$, and $D^{\prime}$ for $\left\{(u, v) \mid u^{2}+v^{2}=1\right\}$. For $D+D^{\prime}$ we use the symbol $\bar{D}$.

We remark that the differential equations are invariant under conformal mappings of the parameter domain. Thus three arbitrarily chosen points $A, B, C$ of $u^{2}+v^{2}=1$ may be mapped into three points $A^{*}, B^{*}, C^{*}$ on $\Gamma$.

2. Preparations for the proof. The most difficult aids we use are already given in [4] and [6]. First of all we need the mentioned

THEOREM OF HeINZ. Let $\Gamma$ be a rectifiable Jordan curve, contained in the unit sphere. Then there is a function $\mathfrak{x}(u, v)$ for $|H|<H_{1}$ which has the properties (A), (B), (C).

Heinz gives the proof for $H_{1}=\left(17^{1 / 2}-1\right) / 8$ in [4, pp. 281-283], but his proof holds also for $H_{1}=1 / 2$, if Satz 1 and Hilfssatz 6 of [6] are used instead of Satz 6 of [4]. The theorem is also a special case of Satz 3 of [6].

Next we give a statement which contains the compactness of surfaces of constant mean curvature.

Lemma 1. Let $\mathfrak{x}^{(n)}(u, v)(n=1,2,3, \cdots)$ be twice continuously differentiable in $D$, satisfy

$$
\Delta \mathfrak{x}^{(n)}=2 H\left(\mathfrak{x}_{u}^{(n)} \times \mathfrak{x}_{\mathfrak{v}}^{(n)}\right)
$$

with $|H|<1$, and assume that $\left|\mathfrak{\mathfrak { r }}^{(n)}(u, v)\right| \leqq 1$ holds in $D$. Then there is a subsequence of $\mathfrak{x}^{(n)}(u, v)$ and a function $\mathfrak{x}(u, v)$ twice continuously differentiable in $D$ with the following properties:

$$
\begin{gathered}
\Delta \mathfrak{x}=2 H\left(\mathfrak{x}_{u} \times \mathfrak{x}_{\mathfrak{v}}\right) \text { and }|\mathfrak{x}(u, v)| \leqq 1 \text { in } D ; \\
\lim _{v \rightarrow \infty} \mathfrak{x}^{\left(n_{v}\right)}(u, v)=\mathfrak{x}(u, v),
\end{gathered}
$$




$$
\begin{aligned}
& \lim _{\nu \rightarrow \infty} \mathfrak{x}_{u}^{\left(n_{\nu}\right)}(u, v)=\mathfrak{x}_{u}(u, v), \\
& \lim _{\nu \rightarrow \infty} \mathfrak{x}_{v}^{\left(n_{\nu}\right)}(u, v)=\mathfrak{x}_{v}(u, v),
\end{aligned}
$$

for $(u, v)$ in $D$; the convergence is uniform in each closed subdomain of $D$.

The proof of Lemma 1 is contained in Hilfssatz 1 and 3 of [4].

Furthermore we use some properties of sequences of monotonic functions which were originally discussed by Helly. We summarize these in a lemma, the proof can be derived from [7].

LEMMA 2. Given a set $s^{(n)}(\phi)$ of strictly monotonic and continuous functions and

$$
\begin{array}{rlrl}
s^{(n)}(\phi+2 \pi) & =s^{(n)}(\phi)+2 \pi, & \\
s^{(n)}(0) & =0 & n=1,2,3, \cdots,-\infty<\phi<+\infty .
\end{array}
$$

Then there exists a function $s(\phi)$ and a subsequence of $s^{(n)}(\phi)$ with the following properties: ties.

(a) $s(\phi)$ is montonic and has at most a denumerable set of discontinui-

(b) In all points where $s(\phi)$ is continuous

$$
\lim _{\nu \rightarrow \infty} s^{\left(n_{\nu}\right)}(\phi)=s(\phi) \text {. }
$$

(c) If

$$
s\left(\phi_{0}\right)=\left\{s\left(\phi_{0}+0\right)+s\left(\phi_{0}-0\right)\right\} / 2
$$

and

$$
d\left(\phi_{0}\right)=\left\{s\left(\phi_{0}+0\right)-s\left(\phi_{0}-0\right)\right\} / 2
$$

then, to each $\delta>0$, there exists $\delta_{1}$ and $n_{1}(\delta)$ such that

$$
\left.\begin{array}{rl}
\left|s(\phi)-s\left(\phi_{0}\right)\right|<d\left(\phi_{0}\right)+\delta, \\
\left|s^{\left(n_{\nu}\right)}(\phi)-s\left(\phi_{0}\right)\right|<d\left(\phi_{0}\right)+\delta
\end{array}\right\} \quad \begin{aligned}
& \text { for }\left|\phi-\phi_{0}\right|<\delta_{1} \\
& \text { and } \nu>n_{1}(\delta) .
\end{aligned}
$$

Finally we state that a solution of (1) is "smooth" if its boundary values are "smooth" and this is just a local property.

Lemma 3. (a) Let $\mathfrak{x}(u, v)$ be a function continuous in $\bar{D}$ and $|\mathfrak{x}| \leqq 1$.

(b) Let the function $\mathfrak{x}(u, v)$ be twice continuously differentiable and satisfy equation (1) with $|H|<1 / 2$ in $D$.

(c) Let the boundary values of $\mathfrak{x}$ be twice continuously differentiable with respect to $\phi$ on $A=\left\{(u, v) \mid u=\cos \phi, v=\sin \phi ; \phi_{-}<\phi<\phi_{+} ; \phi_{-}, \phi_{+}\right.$ fixed . 
Then for each closed subdomain $B$ of $D+A$ there is a number $M$ depending on $B$ and the boundary values on $A$ such that

$$
\mathfrak{x}_{u}^{2}+\mathfrak{x}_{v}^{2} \leqq M^{2}
$$

holds in $B$.

Proof. This lemma is known to be correct for any such domain $B$ if $H=0$, that is for harmonic functions. Using this fact and Hilfssatz 2 and Hilfssatz 3 of [6] the above estimate can be obtained. The method is analogous to that on pages 308-309 of [6].

\section{The proof of the theorem.}

3.1. Let $H$ be a real number with $|H|<1 / 2$. It is no restriction to assume that $H$ is non-negative. The Jordan curve $\Gamma$ may be given by $\mathfrak{y}(t)=(x(t), y(t), z(t))$ continuous in $-\infty<t<+\infty, \mathfrak{y}(t+2 \pi)=\mathfrak{y}(t)$, $|\mathfrak{y}(t)| \leqq 1$. We can find a set of rectifiable Jordan curves $\Gamma_{n}$ represented by $\mathfrak{y}^{(n)}(t)$ continuous in $-\infty<t<+\infty$ and such that $\mathfrak{y}^{(n)}(t+2 \pi)$ $=\mathfrak{y}^{(n)}(t),\left|\mathfrak{y}^{(n)}\right| \leqq 1$ and which for $n \rightarrow \infty$ converges to $\mathfrak{y}(t)$ uniformly in $t$, (for example, use approximations by polygonals without double points). Due to the theorem of Heinz there exist functions $\mathfrak{x}^{(n)}(u, v)$ which represent surfaces of constant mean curvature having the boundary curves $\Gamma_{n}$ respectively. Each of the functions $\mathfrak{x}^{(n)}(u, v)$ generates a topological mapping of $D^{\prime}$ (which may be represented by the parameter $\phi$ ) onto $\Gamma_{n}$ (represented by $t$ ). We can describe these mappings by continuous and monotonic functions $t=s^{(n)}(\phi)$ and we can suppose due to a conformal mapping of the unit circle that $s^{(n)}(0)=0$ (which implies $\left.s^{(n)}(2 \pi)=2 \pi\right), s^{(n)}(1)=1, s^{(n)}(2)=2$. From Lemma 2 we infer that there is a subsequence (and we suppose that it is the whole sequence) which converges to a monotonic function $s(\phi)$ with the described properties. By Lemma 1 another selection yields a subsequence of the $\mathfrak{r}^{(n)}(u, v)$ which is convergent in $D$ together with the first derivatives and the convergence is uniform in each closed subdomain of the unit circle. Let us again suppose that the whole sequence is convergent. Then we have

$$
\begin{gathered}
\lim _{n \rightarrow \infty} \mathfrak{x}^{(n)}(u, v)=\mathfrak{x}(u, v) ; \\
\lim _{n \rightarrow \infty} \mathfrak{x}_{u}^{(n)}(u, v)=\mathfrak{x}_{u}(u, v), \quad \lim _{n \rightarrow \infty} \mathfrak{x}_{\mathfrak{v}}^{(n)}(u, v)=\mathfrak{r}_{v}(u, v)
\end{gathered}
$$

and the limit function satisfies the equations (1) and (2) in $D$.

3.2. Now we prove that the limit function $\mathfrak{x}(u, v)$ has the boundary values $\mathfrak{y}(s(\phi))$ in all points where $s(\phi)$ is continuous. We write $\overline{\mathfrak{y}}(\phi)$ $\equiv \mathfrak{y}(s(\phi)) ; \overline{\mathfrak{y}}^{(n)}(\phi) \equiv \mathfrak{y}^{(n)}\left(s^{(n)}(\phi)\right)$ and 


$$
g\left(\phi_{0}\right)=\max \left|\mathfrak{y}(t)-\overline{\mathfrak{y}}\left(\phi_{0}\right)\right| \text { for }\left|t-s\left(\phi_{0}\right)\right| \leqq d\left(\phi_{0}\right),
$$

furthermore $p(\phi)=(u(\phi), v(\phi))=(\cos \phi$, sin $\phi)$ for points of $D^{\prime}$ and $p_{1}=\left(u_{1}, v_{1}\right)=(r \cdot \cos \vartheta, r \cdot \sin \vartheta)$ for points of $D$.

Given $\epsilon>0$ there exist $\delta>0$ and $n(\epsilon)$ such that

$$
\begin{aligned}
\left|\mathfrak{y}(t)-\overline{\mathfrak{y}}\left(\phi_{0}\right)\right| & <g\left(\phi_{0}\right)+\epsilon, \\
\left|\mathfrak{y}^{(n)}(t)-\overline{\mathfrak{y}}\left(\phi_{0}\right)\right| & <g\left(\phi_{0}\right)+\epsilon
\end{aligned}
$$

for $\left|t-s\left(\phi_{0}\right)\right|<d\left(\phi_{0}\right)+\delta, n \geqq n(\epsilon)$.

According to Lemma $2(\mathrm{c})$ we can find $\delta_{1}$ and $n_{1}(\delta)$ such that

$$
\left|\overline{\mathfrak{y}}^{(n)}(\phi)-\overline{\mathfrak{y}}\left(\phi_{0}\right)\right|<g\left(\phi_{0}\right)+\epsilon
$$

for $\left|\phi-\phi_{0}\right|<\delta_{1}, n \geqq \max \left(n(\epsilon), n_{1}(\delta)\right)$ holds. Due to Hilfssatz 2 of [6] we have the estimate

$$
\begin{aligned}
\left|\overline{\mathfrak{y}}^{(n)}\left(\phi_{0}\right)-\mathfrak{x}^{(n)}\left(p_{1}\right)\right| & \\
\leqq & \frac{H}{2(1-2 H)}\left|\int_{D^{\prime}} K\left(\phi, p_{1}\right)\left(\overline{\mathfrak{y}}^{(n)}(\phi)\right)^{2} d \phi-\left(\overline{\mathfrak{y}}^{(n)}\left(\phi_{0}\right)\right)^{2}\right| \\
& +\frac{1-H}{1-2 H}\left|\int_{D^{\prime}} K\left(\phi, p_{1}\right) \overline{\mathfrak{y}}^{(n)}(\phi) d \phi-\overline{\mathfrak{y}}^{(n)}\left(\phi_{0}\right)\right|
\end{aligned}
$$

where

$$
K\left(\phi, p_{1}\right)=\frac{1}{2 \pi} \frac{1-r^{2}}{\left(1-2 r \cos (\vartheta-\phi)+r^{2}\right)} .
$$

Estimating the two terms on the right side we use the conclusions of potential theory $[2$, p. 244]. There is a number $c(H)>1$ so that the inequality holds

$$
\left|\overline{\mathfrak{y}}^{(n)}\left(\phi_{0}\right)-\mathfrak{x}^{(n)}\left(p_{1}\right)\right| \leqq(c(H)-1)\left(g\left(\phi_{0}\right)+\epsilon+\Delta_{0} / \Delta_{1}^{2}\right)
$$

provided $\left|p_{1}-p\left(\phi_{0}\right)\right|<\Delta_{0}$ and $\left|p_{1}-p(\phi)\right|>\Delta_{1}>0$ for $\phi_{0}+\delta_{1} \leqq \phi \leqq \phi_{0}$ $+2 \pi-\delta_{1}$. Therefore we have

$$
\begin{aligned}
\left|\overline{\mathfrak{y}}\left(\phi_{0}\right)-\mathfrak{x}^{(n)}\left(p_{1}\right)\right| & \leqq\left|\overline{\mathfrak{y}}^{(n)}\left(\phi_{0}\right)-\overline{\mathfrak{y}}\left(\phi_{0}\right)\right|+\left|\overline{\mathfrak{y}}^{(n)}\left(\phi_{0}\right)-\mathfrak{x}^{(n)}\left(p_{1}\right)\right| \\
& \leqq c(H)\left(g\left(\phi_{0}\right)+\epsilon+\Delta_{0} / \Delta_{1}^{2}\right)
\end{aligned}
$$

and for the limit function

$$
\left|\overline{\mathfrak{y}}\left(\phi_{0}\right)-\mathfrak{x}\left(p_{1}\right)\right| \leqq c(H)\left(g\left(\phi_{0}\right)+\epsilon+\Delta_{0} / \Delta_{1}^{2}\right) .
$$

If $s(\phi)$ is continuous at $\phi_{0}$, then $g\left(\phi_{0}\right)=0$ and the above inequality states that $\mathfrak{x}(u, v) \rightarrow \overline{\mathfrak{y}}\left(\phi_{0}\right)$ for $(u, v) \rightarrow p\left(\phi_{0}\right)$.

3.3. It still remains to be proved that $\mathfrak{x}(u, v)$ takes on the boundary 
values $\overline{\mathfrak{y}}(\phi)$ everywhere and that $\mathfrak{y}(\phi)$ is a topological mapping of the unit circle onto $\Gamma$, or equivalently, that $t=s(\phi)$ is continuous and strictly monotonic.

Let us first suppose that $s(\phi)$ is discontinuous in $\phi_{0}$, i.e. $d\left(\phi_{0}\right)>0$, and it is no restriction to assume $\phi_{0}=0$.

Write

$$
\mathfrak{y}_{+}=\overline{\mathfrak{y}}(0+0), \quad \mathfrak{y}_{-}=\overline{\mathfrak{y}}(0-0) .
$$

Then to $\epsilon>0$ there is $\delta>0$ such that

$$
\left|\mathfrak{y}\left(t_{1}\right)-\mathfrak{y}\left(t_{2}\right)\right|<\epsilon \text { provided }\left|t_{1}-t_{2}\right|<\delta .
$$

To $\delta>0$ there exists $\delta_{1}>0$ such that, due to Lemma 2 , the inequality

$$
s(0+0) \leqq s(\phi) \leqq s(0+0)+\delta \text { for } 0<\phi<\delta_{1} \text { holds. }
$$

Therefore $g(\phi) \leqq \epsilon$ for such values of $\phi$. From (4) we conclude that to each $p(\phi)$ there is an open neighborhood $U(\phi)$ in $D$ given by

$$
U(\phi)=\left\{p_{1}\left|p_{1} \in D,\right| p_{1}-p(\phi) \mid<\Delta_{0}(\phi)\right\}
$$

such that

$$
\left|\mathfrak{x}\left(p_{1}\right)-\overline{\mathfrak{y}}(\phi)\right|<3 \cdot c(H) \cdot \epsilon, \quad p_{1} \in U(\phi),
$$

or

$$
\left|\mathfrak{x}\left(p_{1}\right)-\mathfrak{y}_{+}\right|<4 \cdot c(H) \cdot \epsilon .
$$

Similarly we get

$$
\left|\mathfrak{x}\left(p_{1}\right)-\mathfrak{y}_{-}\right|<4 \cdot c(H) \cdot \epsilon, \quad p_{1} \in U(\phi),-\delta_{1}<\phi<0 .
$$

We write $z=u+i v$ and transform the parameter by

$$
\frac{w-1}{w+1}=\lambda \frac{z-1}{z+1}
$$

$\lambda$ positive.

Obviously the unit circle is mapped onto itself. We define $\mathfrak{x}_{\lambda}(u, v)$ $=\mathfrak{r}(w)$ and choose $\lambda$ so that the arc $\left(0, \delta_{1}\right)$ of the unit circle is mapped onto an arc which contains $1 \leqq \phi \leqq 2$ in its interior. (The arc $\left(0,-\delta_{1}\right)$ is mapped onto an arc which contains $(-2 \leqq \phi \leqq-1)$.)

It is easily seen that the maps of the $U(\phi)$ cover a whole strip along $1 \leqq \phi \leqq 2$, or $(-2 \leqq \phi \leqq-1)$, in $D$. Therefore

$$
\left|\mathfrak{x}_{\lambda}\left(p_{1}\right)-\mathfrak{y}_{+}\right| \leqq 4 \cdot c(H) \cdot \epsilon
$$

for $p_{1}=\left(r_{0} \cos \phi, r_{0} \sin \phi\right), 1 \leqq \phi \leqq 2$ and fixed $r_{0}<1$ sufficiently near to 1 , and 


$$
\left|\mathfrak{x}_{\lambda}\left(p_{1}\right)-\mathfrak{y}_{-}\right| \leqq 4 \cdot c(H) \cdot \epsilon
$$

for $p_{1}=\left(r_{0} \cos \phi, r_{0} \sin \phi\right),-1 \geqq \phi \geqq-2$.

While the boundary values of $\mathfrak{x}_{\lambda}(u, v)$ can be discontinuous, $z_{\lambda}(u, v) \equiv \mathfrak{x}_{\lambda}\left(r_{0} u, r_{0} v\right)$ is continuous in $D$, satisfies equations (1) and (2) and

$$
\begin{aligned}
& \left|z_{\lambda}(p(\phi))-\mathfrak{y}_{+}\right|<4 \cdot c(H) \cdot \epsilon, \quad 1 \leqq \phi \leqq 2, \\
& \left|z_{\lambda}(p(\phi))-\mathfrak{y}_{-}\right|<4 \cdot c(H) \cdot \epsilon \quad-2 \leqq \phi \leqq-1 .
\end{aligned}
$$

Corresponding to a null sequence $\epsilon^{(1)}, \epsilon^{(2)}, \ldots$ we get functions $z_{\lambda}^{(1)}(u, v), z_{\lambda}^{(2)}(u, v), \cdots$ with the above properties.

Due to Lemma 1 there exists a convergent subsequence and the limit vector $z(u, v)$ again satisfies the equations (1) and (2) and $|z| \leqq 1$. The functions satisfy the assumptions of Hilfssatz 2 of [6]. Therefore applying the method of 3.2 it can be shown that $z(u, v)$ takes on the boundary values $\mathfrak{y}_{+}$on $A_{+}=\{p(\phi) \mid 1<\phi<2\}$ and $\eta_{-}$on $A_{-}=\{p(\phi) \mid-2<\phi<-1\}$. Applying Lemma 3 (using $A_{+}$for $A$ ), we see that for a subdomain $B \in D+A$, the first derivatives of $z(u, v)$ are bounded. Let $B \cap A_{1}=A^{*}$ be not empty, containing at least two points. Then we extend the function $z(u, v)$ by reflection on $A^{*}$ and infer that $z(u, v)$ is analytic in the extended region. Equations $(2)$ and $z(u, v)=\mathfrak{y}_{+}$on $A^{*}$ show that $z(u, v)=\mathfrak{y}_{+}$is constant in this region and therefore, due to the analytic character, $z(u, v)$ is constant in $D$. This however contradicts the fact that $z(u, v)$ has the boundary value $\mathfrak{y}_{-} \neq \mathfrak{y}_{+}$on $A_{-}$. Therefore the function $s(\phi)$ has to be continuous.

If we now suppose that $s(\phi)$ is not strictly monotonic, then there is an interval $\phi_{-}<\phi<\phi_{+}$where $s(\phi)$ is constant. This implies that $\mathfrak{x}(u, v)$ has constant boundary values along an arc of $D^{\prime}$. As we just proved, this infers $\mathfrak{x}(u, v)$ is constant.

But we assumed $s(1)=1, s(0)=0$ and we proved that $s(\phi)$ is continuous. Therefore $\mathfrak{y}(s(1))=\mathfrak{y}(1) \neq \mathfrak{y}(s(0))=\mathfrak{y}(0)$ and, as was also shown in $3.2, \mathfrak{x}(u, v)$ takes on these boundary values. Therefore $\mathfrak{x}(u, v)$ cannot be constant.

This contradiction proves that $s(\phi)$ is strictly monotonic, which completes the proof of Theorem $1^{\prime}$.

4. Remarks. Due to a conformal mapping we suppose the domain of $z(u, v)$ to be the upper half plane. Using the method of 3.3 we can conclude that $z(u, v)$ has a constant value $\mathfrak{y}_{+}$for $0<u$ and a value $\mathfrak{y}_{-} \neq \mathfrak{y}_{+}$for $u<0$. We can explicitly write down a solution of

$$
\Delta \mathfrak{r}=2 H\left(\mathfrak{r}_{u} \times \mathfrak{r}_{v}\right)
$$


with these boundary values, namely

$$
\mathfrak{r}(u, v)=\mathfrak{y}_{+}+\pi^{-1}\left(\mathfrak{y}_{-}-\mathfrak{y}_{+}\right) \arctan (v / u), \quad 0 \leqq \arctan (v / u) \leqq \pi
$$

and it is obvious, that the conditions

$$
\mathfrak{x}_{u}^{2}=\mathfrak{x}_{v}^{2} \quad \text { and } \quad\left(\mathfrak{x}_{u}, \mathfrak{x}_{v}\right)=0
$$

are not satisfied for all $u, v$. But the open question is whether we have other solutions of this boundary value problem or not. The trouble is that the Dirichlet integral of $\mathfrak{x}(u, v)$ is infinite and is infinite also for any other solution of the above equations. Therefore the uniqueness proof contained in $[4$, Satz 5$]$ or $[6$, p. 317] is not applicable.

\section{BIBLIOGRAPHY}

1. R. Courant, Dirichlet's principle, conformal mapping and minimal surfaces, New York, Interscience, 1950.

2. R. Courant and D. Hilbert, Methoden der mathematischen Physik, vol. 2, Berlin, Springer-Verlag, 1937.

3. J. Douglas, Solution of the problem of Plateau, Trans. Amer. Math. Soc. vol. 33 (1931) pp. 263-321.

4. E. Heinz, Ueber die Existenz einer Flaeche konstanter mittlerer Kruemmung bei vorgegebener Berandung, Math. Ann. vol. 127 (1954) pp. 258-287.

5. - On the existence problem for surfaces of constant mean curvature, Comm. Pure Appl. Math. vol. 9 (1956) pp. 467-470.

6. H. Werner, Das Problem von Douglas fuer Flaechen konstanter mittlerer Kruemmung, Math. Ann. vol. 133 (1957) pp. 303-319.

7. A. Wintner, Spektraltheorie der unendlichen Matrizen, Leipzig, 1929.

University of Southern California 